\title{
Antibiofilm Synergy of $\beta$-Lactams and Branched Polyethylenimine against MRSE \& MRSA
}

Anh K. Lam, Cassandra Wouters, Erika Moen, Jennifer Pusavat, Hannah Panlilio, and Prof. Charles V. Rice The University of Oklahoma, Department of Chemistry and Biochemistry, Stephenson Life Sciences Research Center

101 Stephenson Parkway, Norman, Oklahoma 73019

\section{Abstract}

Microbial biofilms are ubiquitous in nature, and they pose a serious threat to public health. Staphylococcus epidermidis and $S$. aureus is the most common clinical isolate from healthcareand medical device-related biofilm infections. No antibiotic currently on the market can eradicate pathogenic biofilms, which contain complex defense mechanisms composed of slime-like extracellular polymeric substances (EPS). Understanding the need to develop alternative approaches, we examine 600-Da branched polyethylenimine (BPEI) against methicillin-resistant Staphylococcus epidermidis (MRSE) and S. aureus (MRSA) biofilms. Here, a microtiter biofilm model is used to test the synergistic effects between the two components of our combination treatment: BPEI and $\beta$-lactam antibiotics. Electron microscopy was used to confirm the growth of crystal violet assays, and biofilm kill curves suggest that BPEl exhibits antibiofilm activity and can potentiate $\beta$-lactams to eradicate MRSE/MRSA biofilms.

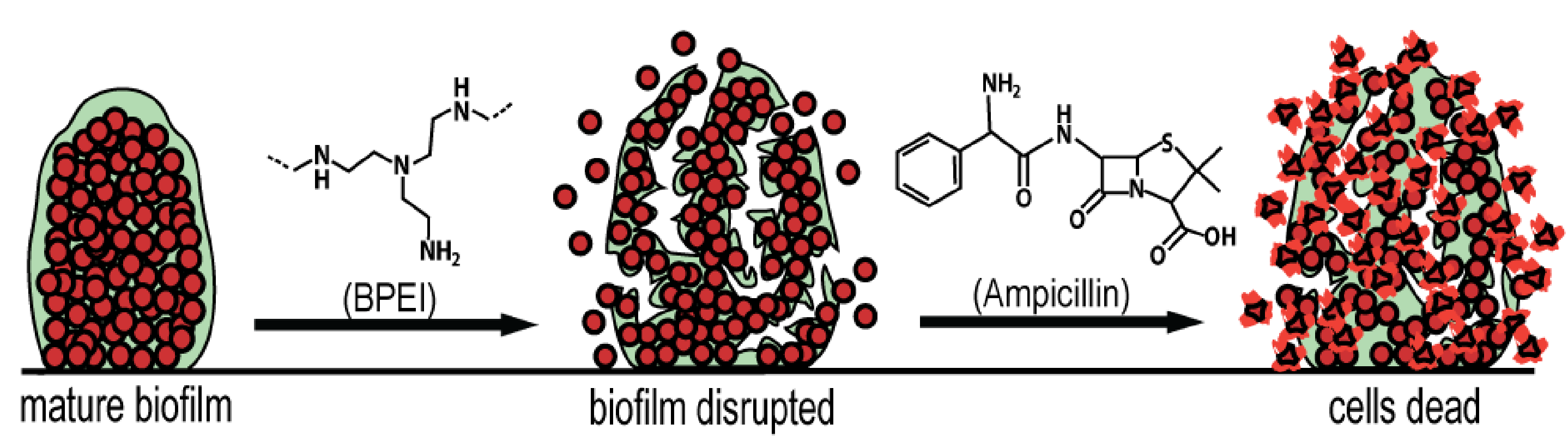

Figure 1. Biofilm-disrupting ability of BPEI. BPEI disrupts established biofilm by collapsing the structure of EPS,
releasing the cells into planktonic stage so that $\beta$-lactam antibiotics can actively target and eradicate them.

Introduction

Bacterial biofilms that are impenetrable to antibiotics pose an even greater threat when they are created by drug resistant bacteria, such as methicilin-resistant $S$. epidermidis \& $S$. aureus (MRSEMRSA). MRSA, MRSE, and her biollms lead to chronic Wound ilfeclions (.e. wound that have not proceeded through a reparative process in three months) that affect millions of Americans each year. With a dwinding arsenal of new antibiotics, existing drugs and regimens must be coupled with potentalors and re valualed as combination treatments for biofilms and antibiotic-lesistant diseases. Our lab previously examined the efficacy of branched polantonic MRSEMRSA a caria Caponic BPEl, with many primary and shoon indirectly targets the resistant factor PBP2a by elctrostatically binding to anionic wall tichoic acid required for PBP2a activity. In this manner, BPEl disabled resistance in MRSEMRSA strins, restoring their susceptibility to traditional $\beta$-actam antibitics. In this study, we futher investigate the application of our technology to MRSE/MRSA bifilms where the EPS contins numerous anionic biomacromolecules. The EPS creates a hydrophobic barrier that increases the tolerance of bacteria towards antimicrobials by reducing or preventing the antibiotics from reaching their target(s). When BPEI binds to EPS, two changes will occur. First, the anionic character of the biofilm will be neutralized leading to a disruption of the EPS matrix. Secondly, because BPEl is a hydrophilic polymer, it will disrupt hydrophobic barriers that hinder diffusion of aqueous antibiotics. Thus, BPEl functions as a potentiator by inhibiting and disrupting the EPS matrix allowing antibiotics to enter and kill the bacteria.

Experimental Methods

Experimental procedures were followed the methods of Lam et al.

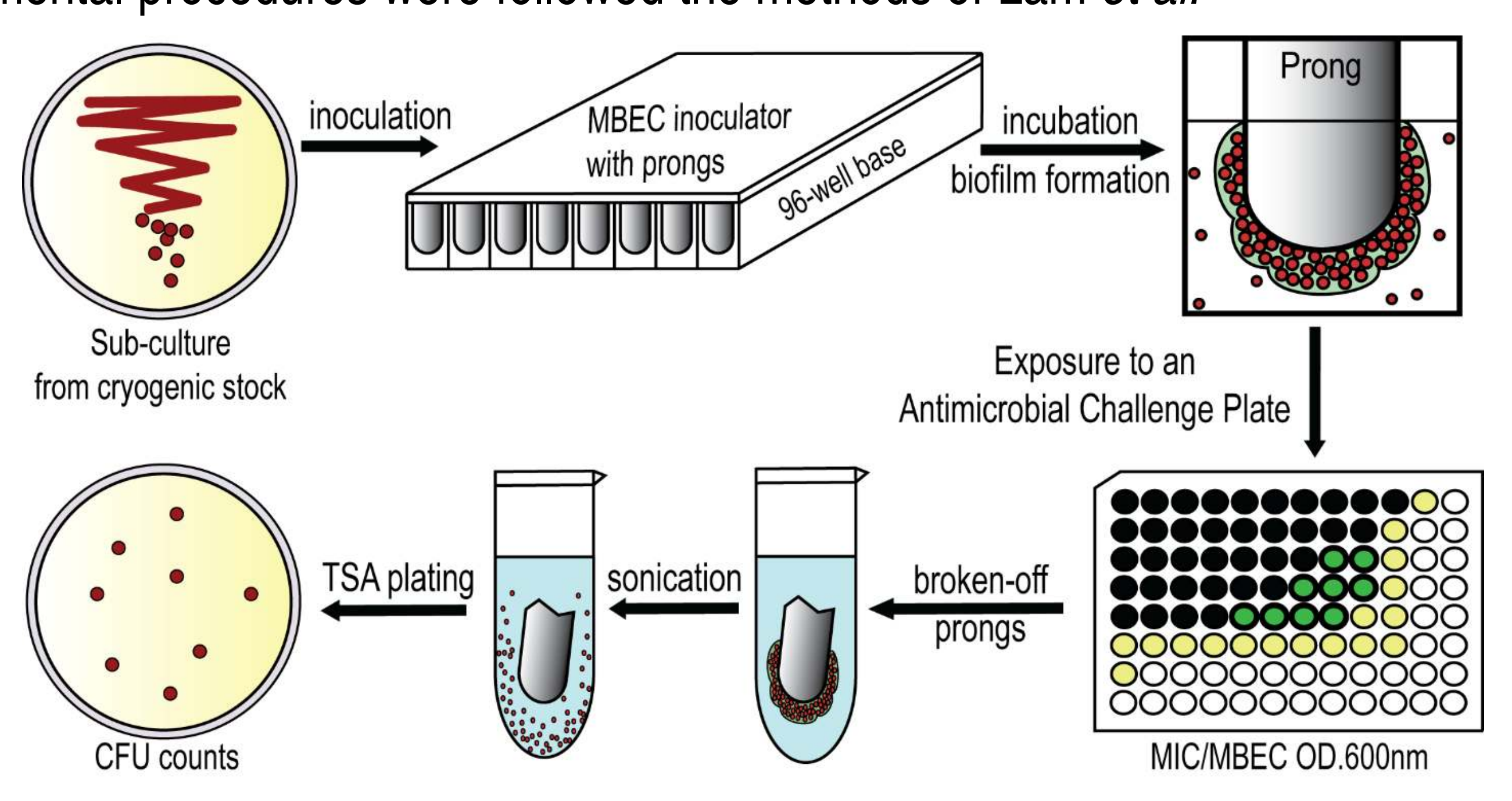

Hgere 2. A schemaicic expertmental procedure of our microfiter biofilim model tor synergistic effect
Results and Discussion
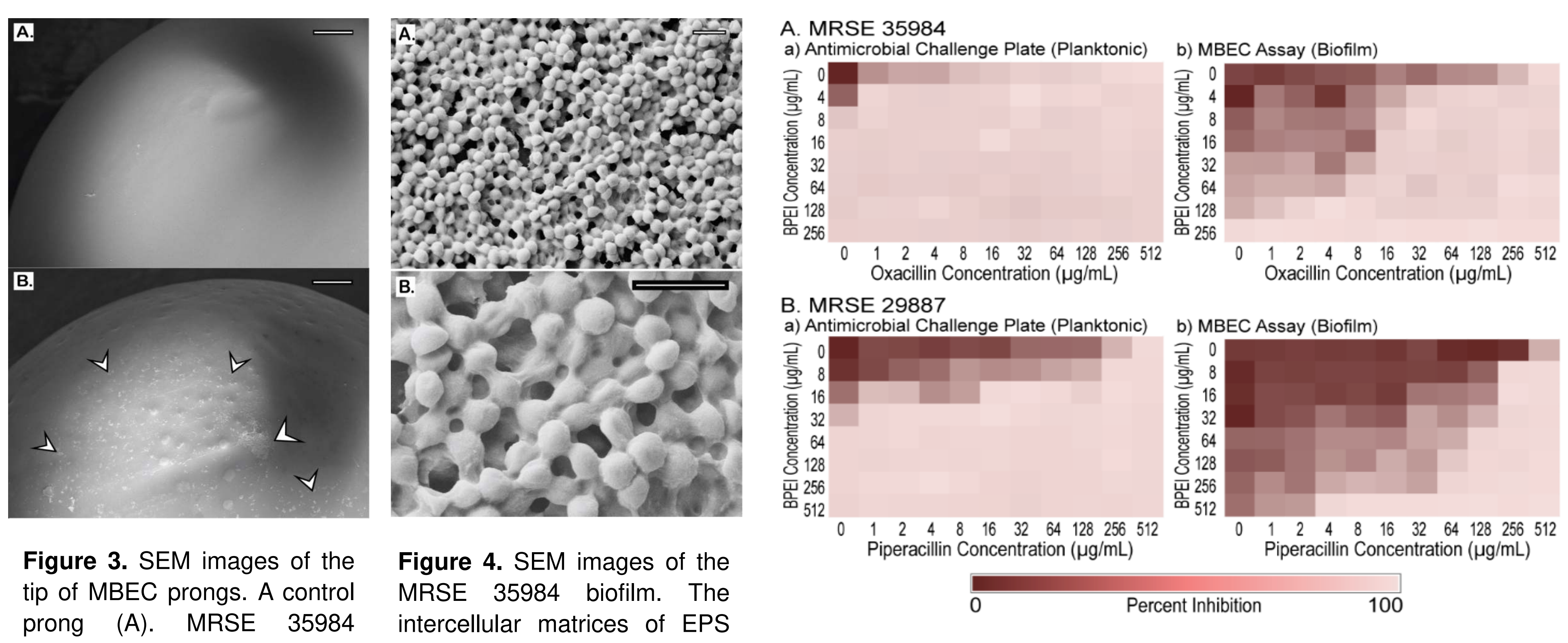

$\begin{array}{ll}\text { Figure 3. SEM images of the } & \text { Figure 4. SEM images of the } \\ \text { tip of MBEC prongs. A control } & \text { MRSE } 35984 \text { biofilm. The }\end{array}$

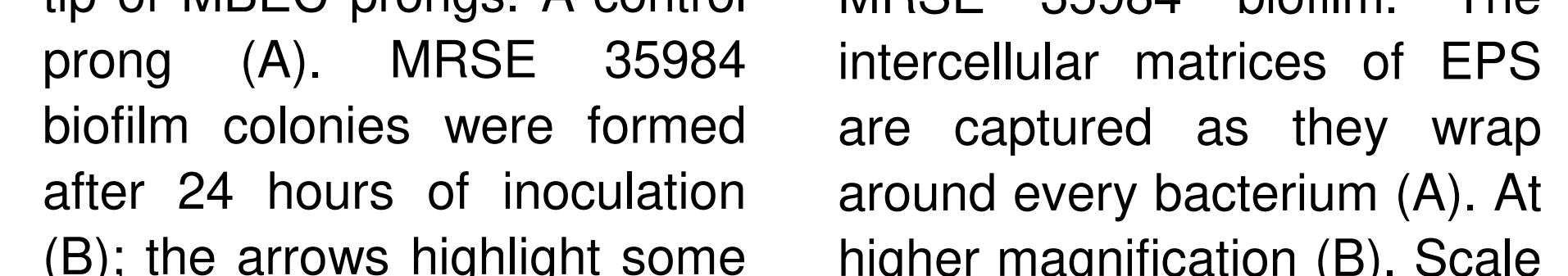

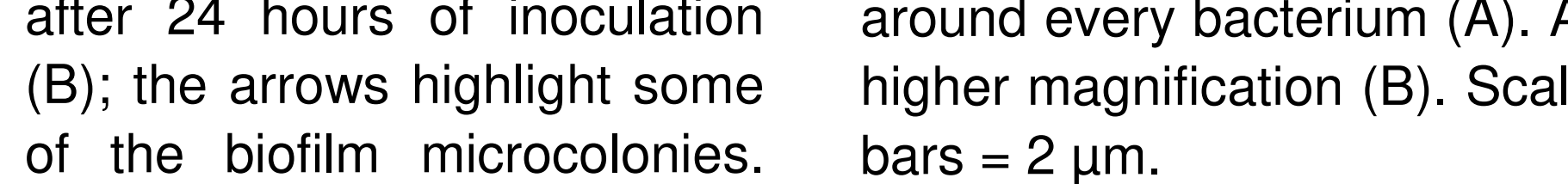
A.

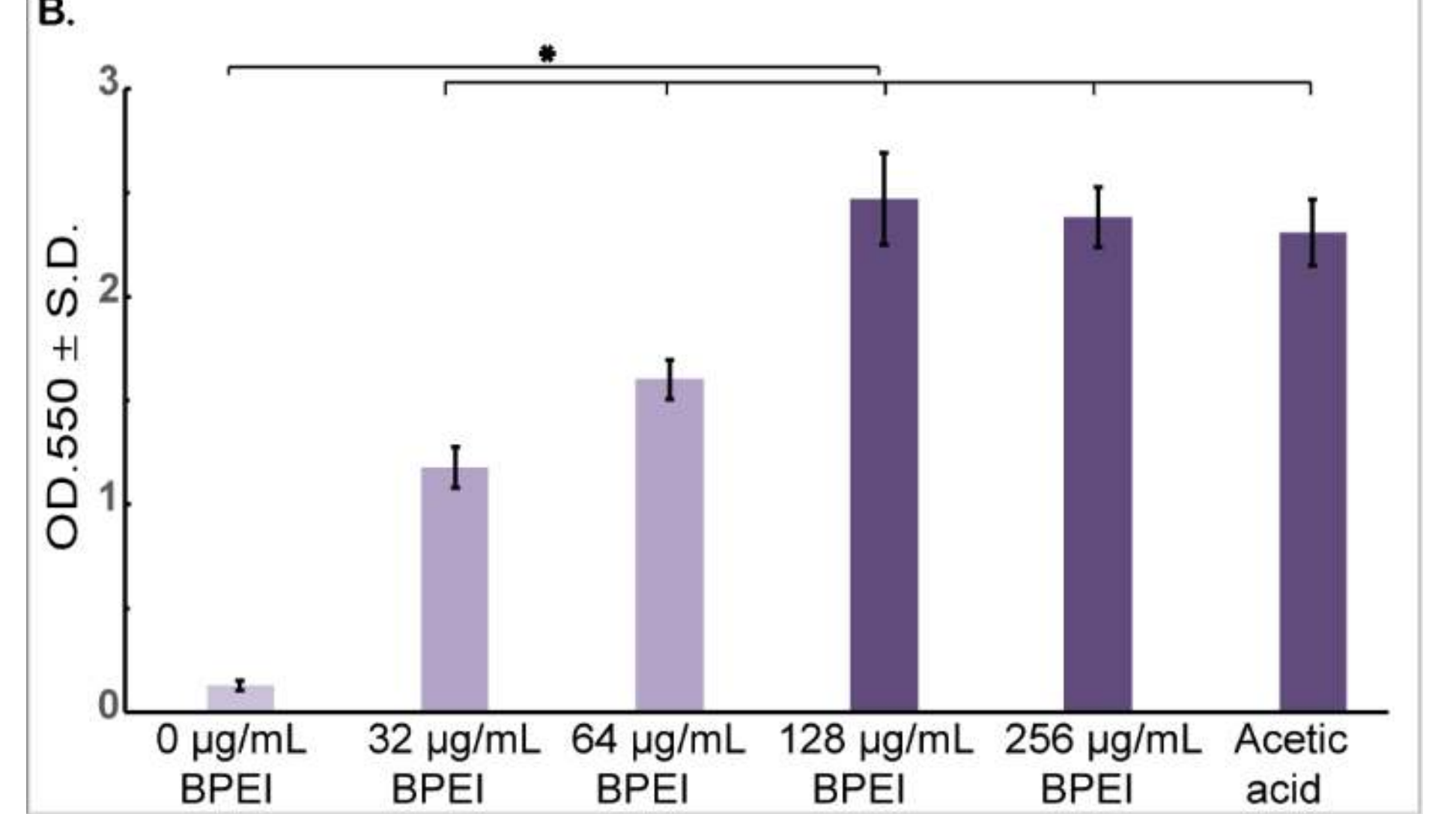

Figure 6. Established MRSE 35984 biofilims stained with crys

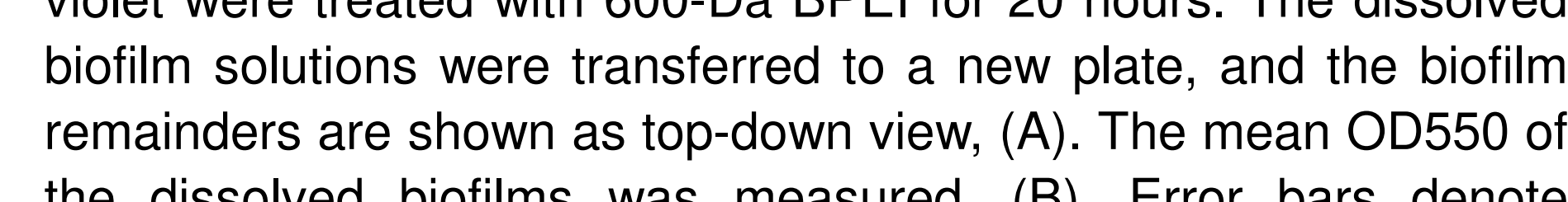

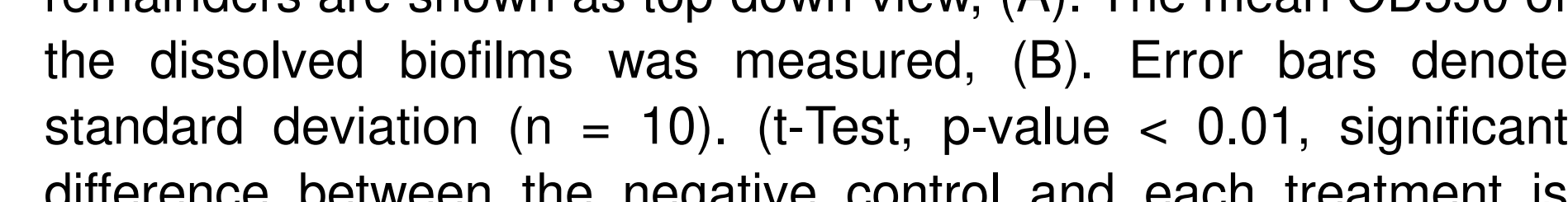
difference between the ne.

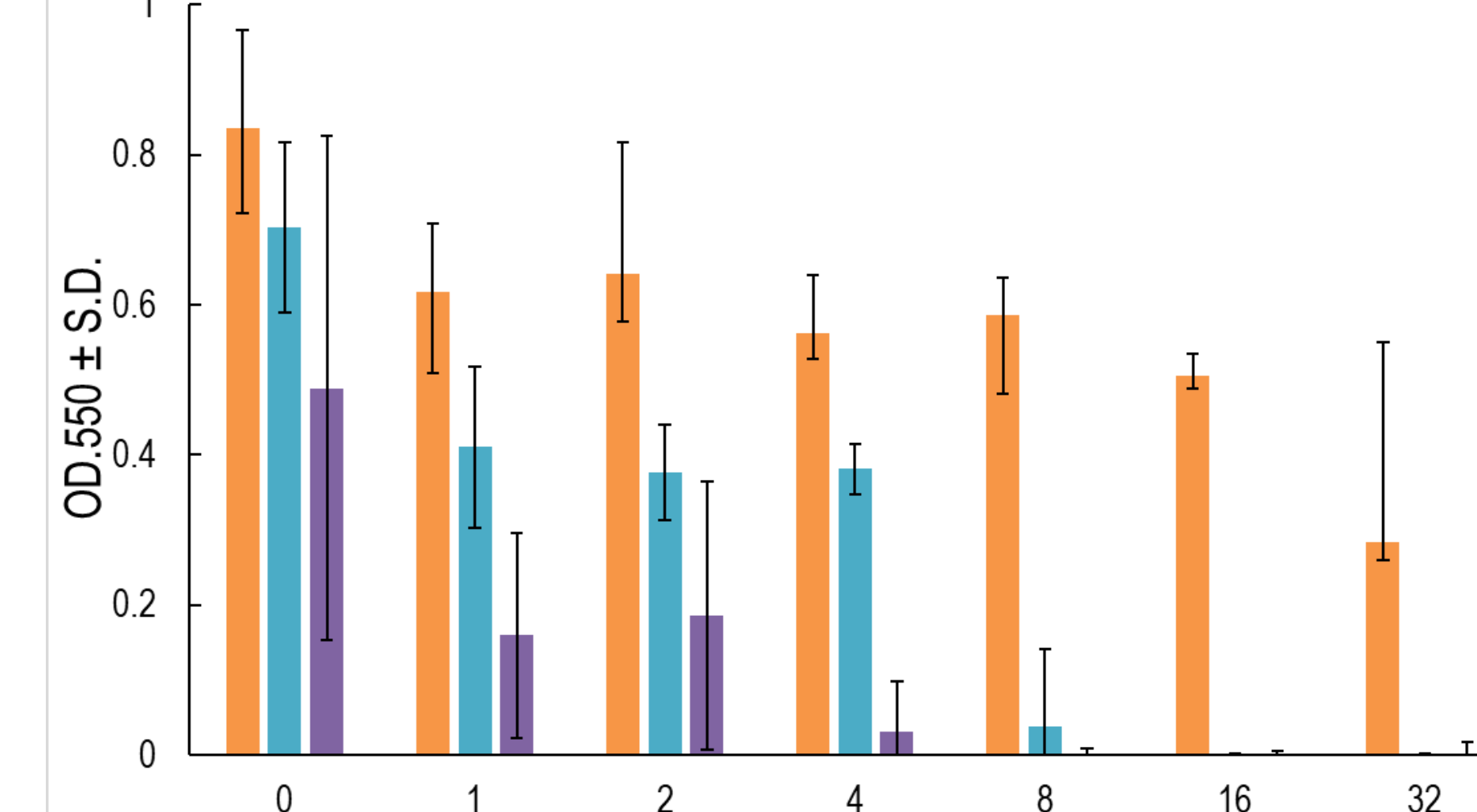

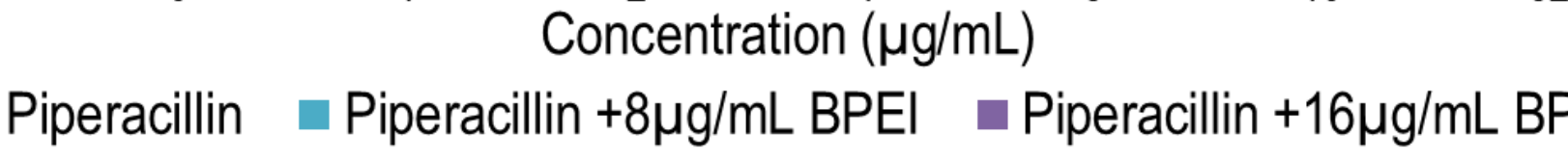
Figure 8. Strong antibiofilim formation synergy between BPEI and
piperacillin was observed, compared to indivivual piperacilinin or

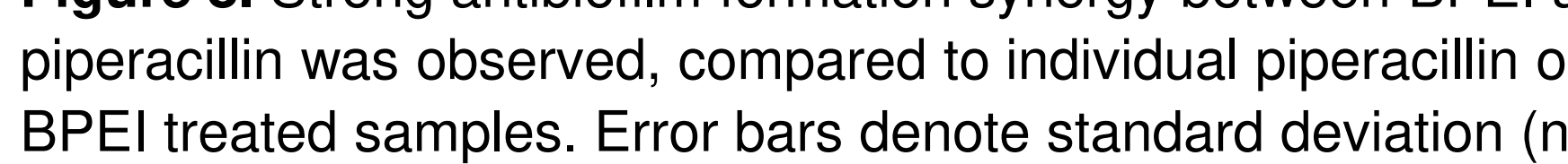

Acknowledgements

Figure 5. Synergisicic effects of BPEE and antibiticics against MRSE 35984
(A) and MRSE 29887 (B) on a 96 -well checkerboard pattern. The synergy
was seen both on the planktonic challenge plates (Aa and Ba) and the 作

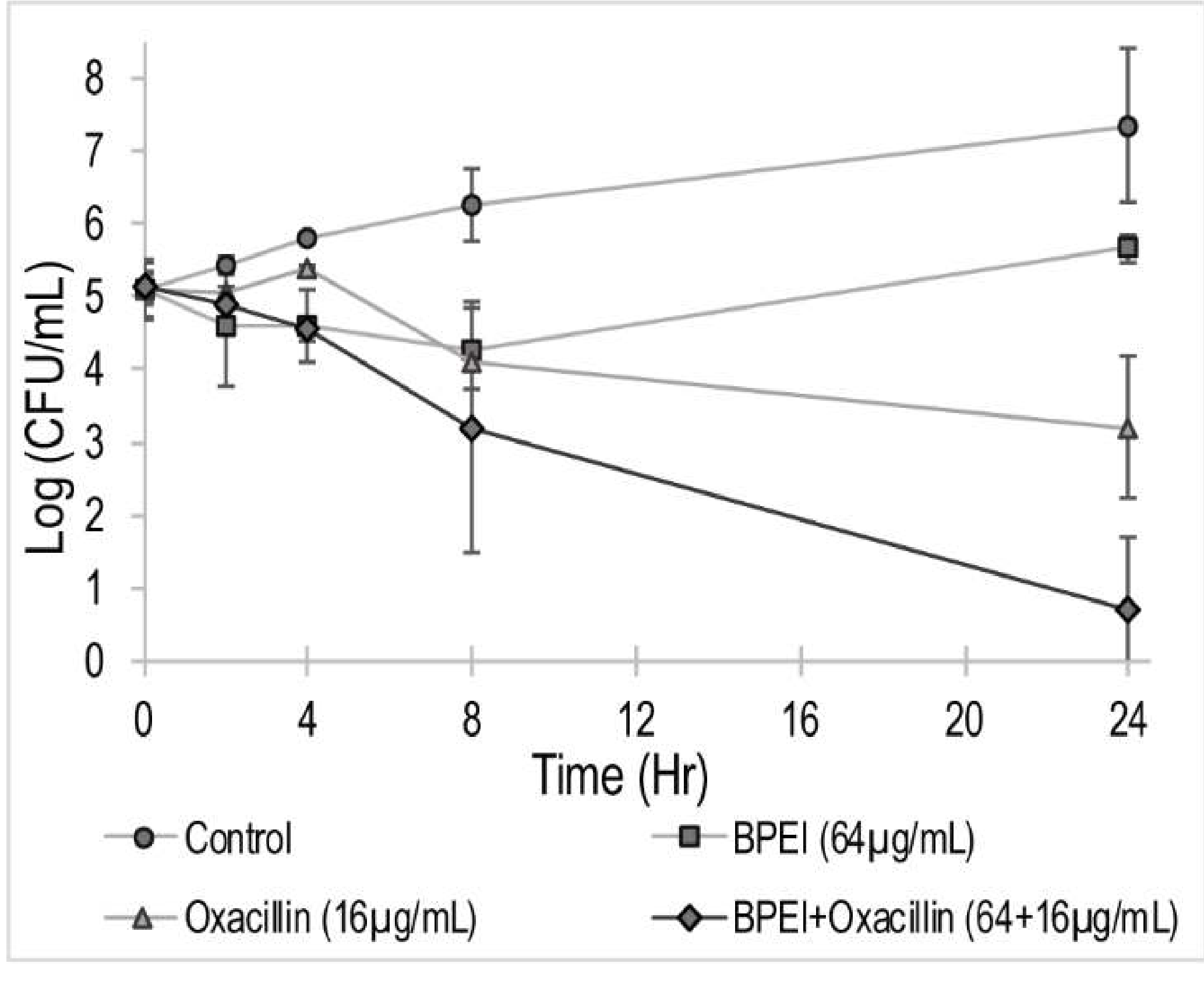

Figure 7. Biofilim kill curve of MRSE 35984 . Only the combination

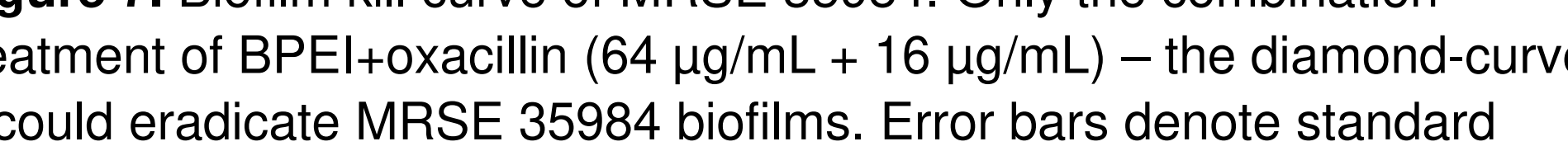
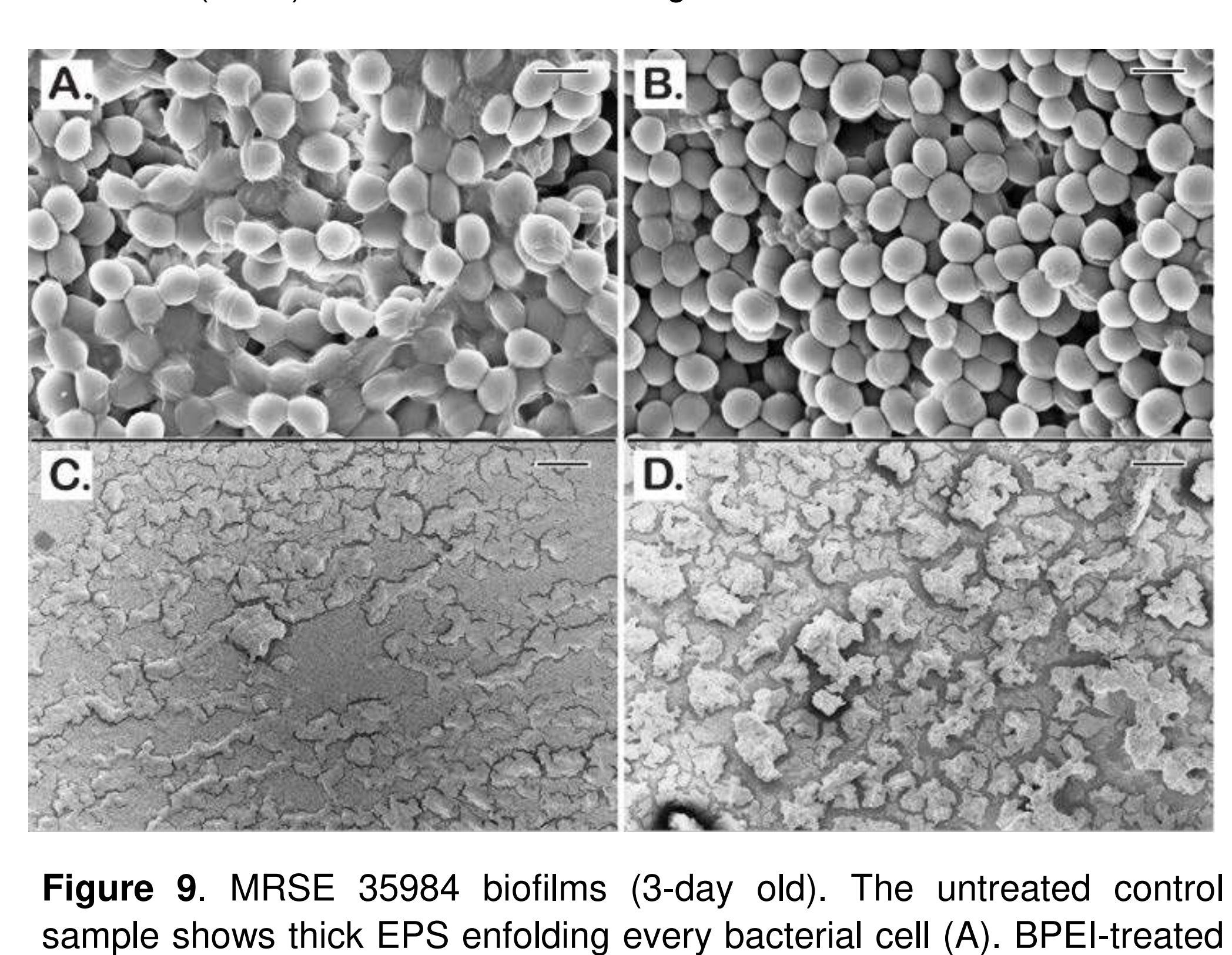
sample shows disrupted EPS and siginiticant number of exposed cell
without the EPS (B). At lower magninication, the untreated cont cel

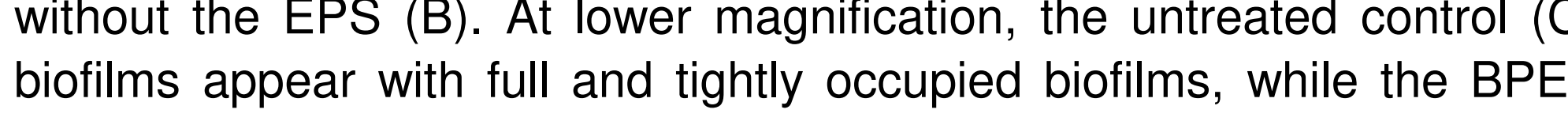

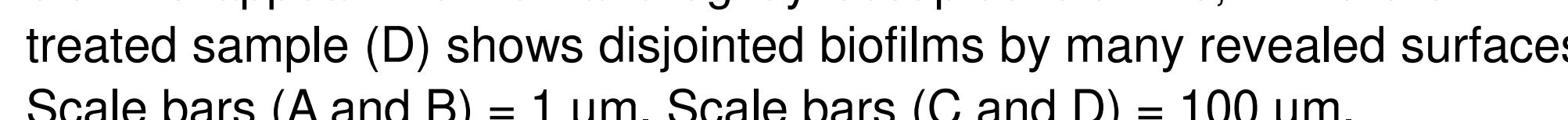

The work in this poster is possible due to the kindness and contributions from Daniel Glatzhofer, PhD., Robert Cichewicz, PhD., Preston Larson, PhD, Robert Brennan, $\mathrm{PhD}$, and all the members in Rice's Lab. We also wan Fin

Science and Technology, National Institutes of Health, and The University of Oklahoma.

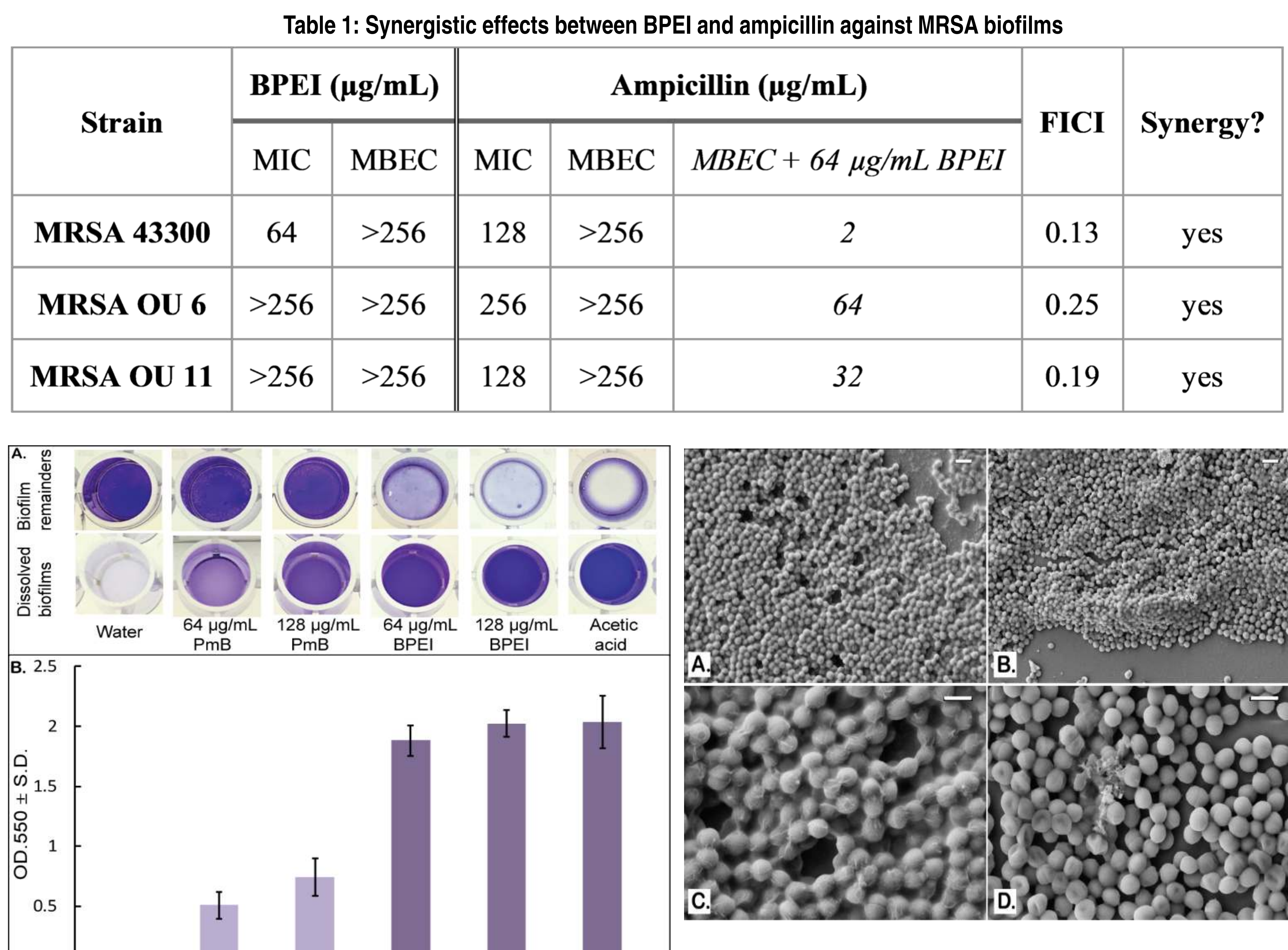

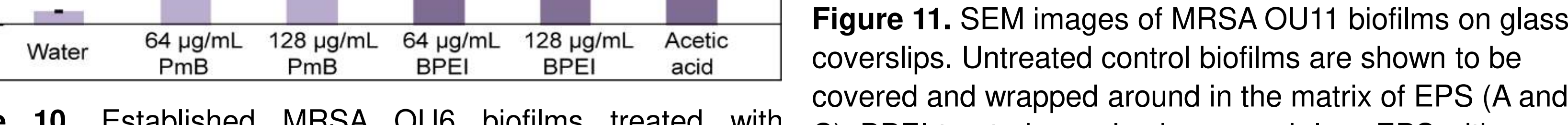

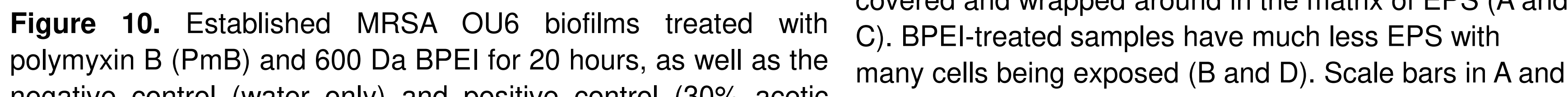
egative control (water only) and positive control $(30 \%$ a a acid). The dissolved biffilm solutions were transferred to a new
plate, and the biofilim remainders are shown as top-down views plate, and the biofilim remainders are shown as top-down view
(A). The mean 00550 of the dissolved biofilim solution was

What's next?!

Broadening the antimicrobial spectrum of BPEl to potentiate non- $\beta$-lactams and against drug-resistant Gram-negative bacteria (Pseudomonas aeruginosa and $E$. coll)

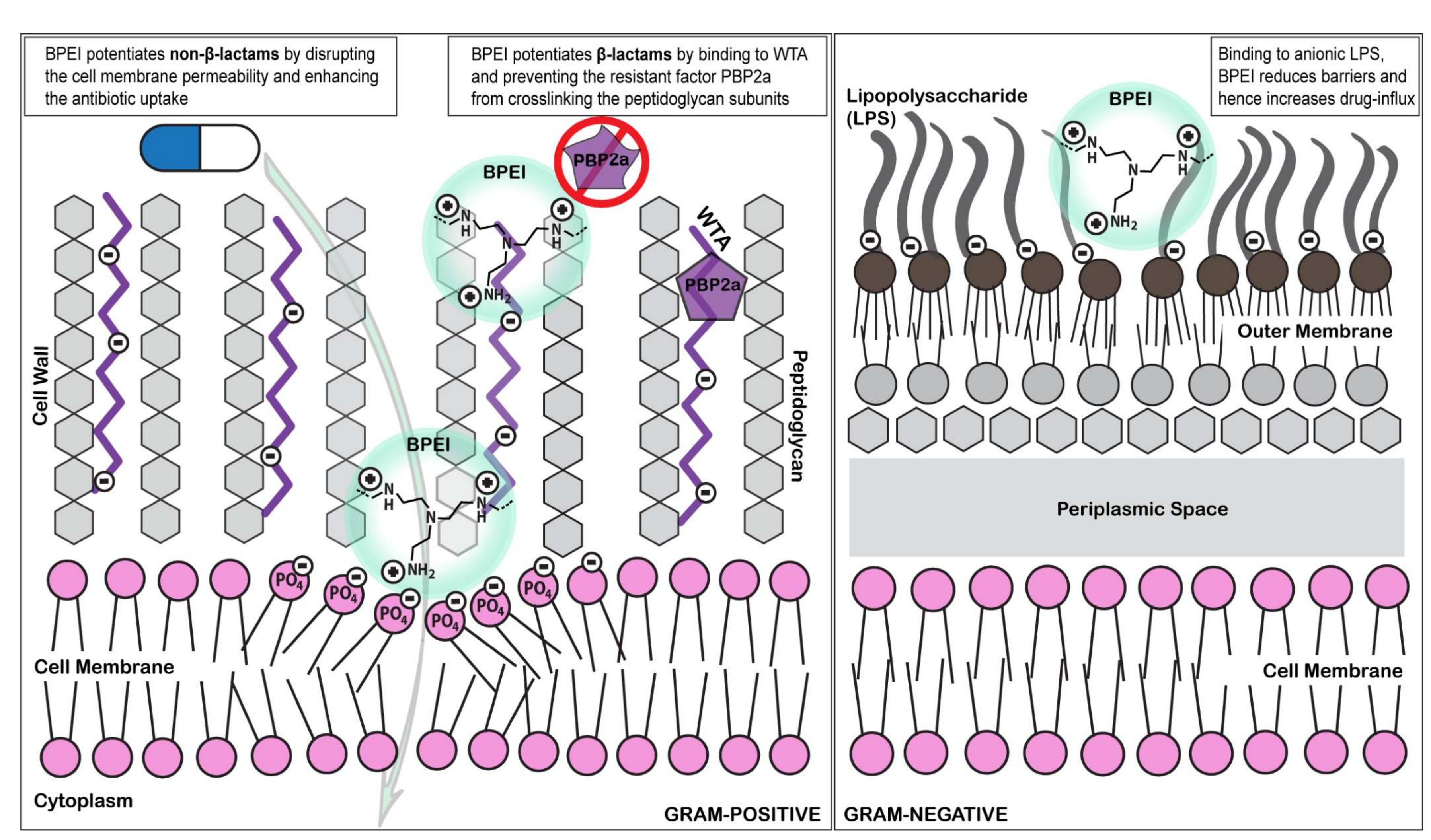

References

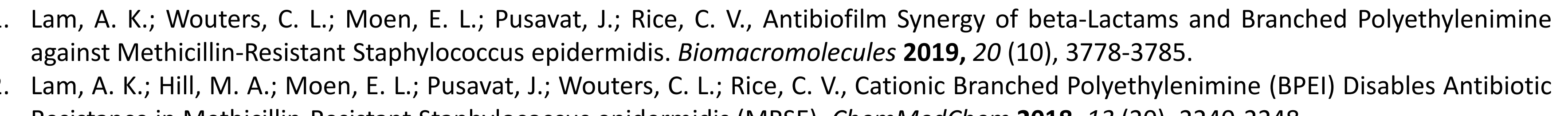

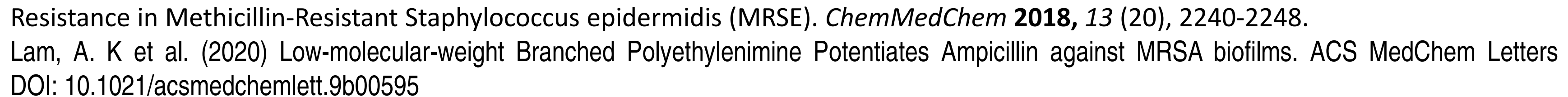

